\title{
Genome-Wide Selective Signatures Reveal Candidate Genes Associated with Hair Follicle Development and Wool Shedding in Sheep
}

\author{
Zhihui Lei ${ }^{1,2,+}$, Weibo Sun ${ }^{1,+}{ }^{\mathbb{C}}$, Tingting Guo ${ }^{1}$, Jianye $\mathrm{Li}^{1}{ }^{1}$, Shaohua Zhu ${ }^{2}$, Zengkui Lu ${ }^{1} \mathbb{D}$, Guoyan Qiao ${ }^{1}$, \\ Mei Han ${ }^{1}$, Hongchang Zhao ${ }^{1}$, Bohui Yang ${ }^{1}$, Liping Zhang ${ }^{2}$, Jianbin Liu ${ }^{1}{ }^{1}$, Chao Yuan ${ }^{1}$ and Yaojing Yue ${ }^{1, *}$ \\ 1 Lanzhou Institute of Husbandry and Pharmaceutical Sciences, Chinese Academy of Agricultural Sciences, \\ Lanzhou 730050, China; lzh887246@163.com (Z.L.); swb887246@126.com (W.S.); guotingting@caas.cn (T.G.); \\ lijianye218@163.com (J.L.); luzengkui@caas.cn (Z.L.); qhqiaogy@163.com (G.Q.); 82101186189@caas.cn (M.H.); \\ 18837101296@163.com (H.Z.); yangbh2004@163.com (B.Y.); liujianbin@caas.cn (J.L.); yuanchao@caas.cn (C.Y.) \\ 2 College of Animal Science and Technology, Gansu Agricultural University, Lanzhou 730070, China; \\ zhu87932890@126.com (S.Z.); zhanglp512@163.com (L.Z.) \\ * Correspondence: yueyaojing@126.com \\ + Zhihui Lei and Weibo Sun both authors contributed equally to this work.
}

\section{check for}

updates

Citation: Lei, Z.; Sun, W.; Guo, T.; Li, J.; Zhu, S.; Lu, Z.; Qiao, G.; Han, M.; Zhao, H.; Yang, B.; et al. Genome-Wide Selective Signatures Reveal Candidate Genes Associated with Hair Follicle Development and Wool Shedding in Sheep. Genes 2021, 12, 1924. https://doi.org/10.3390/ genes12121924

Academic Editor: Brenda Mae Murdoch

Received: 13 October 2021

Accepted: 27 November 2021

Published: 29 November 2021

Publisher's Note: MDPI stays neutral with regard to jurisdictional claims in published maps and institutional affiliations.

Copyright: (c) 2021 by the authors. Licensee MDPI, Basel, Switzerland. This article is an open access article distributed under the terms and conditions of the Creative Commons Attribution (CC BY) license (https:/ / creativecommons.org/licenses/by/ $4.0 /)$.

\begin{abstract}
Hair follicle development and wool shedding in sheep are poorly understood. This study investigated the population structures and genetic differences between sheep with different wool types to identify candidate genes related to these traits. We used Illumina ovine SNP 50K chip genotyping data of 795 sheep populations comprising 27 breeds with two wool types, measuring the population differentiation index (Fst), nucleotide diversity ( $\theta \pi$ ratio), and extended haplotype homozygosity among populations (XP-EHH) to detect the selective signatures of hair sheep and fine-wool sheep. The top $5 \%$ of the Fst and $\theta \pi$ ratio values, and values of $\mathrm{XP}-\mathrm{EHH}<-2$ were considered strongly selected SNP sites. Annotation showed that the PRX, SOX18, TGM3, and TCF3 genes related to hair follicle development and wool shedding were strongly selected. Our results indicated that these methods identified important genes related to hair follicle formation, epidermal differentiation, and hair follicle stem cell development, and provide a meaningful reference for further study on the molecular mechanisms of economically important traits in sheep.
\end{abstract}

Keywords: sheep; SNP chip; selective signatures; Fst; $\theta \pi$ ratio; $\mathrm{XP}-\mathrm{EHH}$

\section{Introduction}

Hair sheep is a kind of sheep with wool shedding that can adapt to a variety of climatic conditions and ecological environment. Hair sheep have coats with few woolly fibers or fibers that are shed annually. Not only do they not have to shear their wool often, but they are more resistant to parasites and diseases. While all sheep technically have both wool and hair fibers, hair sheep breeds have higher proportions of the latter. Nevertheless, since the breeds are so adaptable, they can thrive in cold climates, too, as they grow thicker undercoats when they move further north [1]. Due to the obvious depilation characters and high anti-parasite [2], hair sheep are becoming popular in the United States and other temperate regions, such as the Dorper, Wiltshire Horn, Katahdin sheep, and Barbados Black belly. The Dorper sheep is a heavy muscled sheep breed that originated in South Africa. It is a result of the breeding between Dorset Horn rams with Blackhead Persian ewes [3]. Dorper sheep breed has two distinct types the White Dorper and the traditional Dorper. The White Dorper is as its name implies a pure white-haired sheep and the traditional Dorper has a white body and black head much lie the Blackhead Persian breed. They are one of the most popular breeds of sheep in the United States today due to their shedding characteristics and superior conformation. The Barbados Black belly sheep is a breed originated in the Caribbean islands and is famous for its parasite resistance and 
cold tolerance [1]. Compared with wool, mutton has become the main economic source of farmer, furthermore, the labor cost required for shearing in the feeding process is higher than the value of wool itself. Therefore, in order to reduce breeding costs, people have a strong interest in sheep varieties with natural depilation $[4,5]$. The purpose is to reduce the feeding cost and increase the economic income of sheep on the basis of maintaining superior characteristics, such as growth rate, fertility and disease resistance. Compared with pure-bred hair sheep, their hybrid offspring can grow more wool fibers but still have the traits of natural shedding [6,7]. Although the main factors affecting wool shedding in this sheep are not clear, there is preliminary evidence that the shedding is strongly controlled by heredity, most of its expression is a single dominant autosomal gene [5]. Therefore, it would be of interest to explore the genetic mechanisms of wool shedding using selective signatures to identify genes related to sheep hair follicle development and the control of wool shedding and to understand their functions.

With the development of high-throughput phenotyping technology and molecular markers, the use of selective signatures can identify the imprints left in the genome during selection [8]. Among many selective signatures analysis methods, the most commonly used include Fst based on the population differentiation index. When the difference in the frequency of the same allele in different populations is greater than the expectation that the two populations are in neutral conditions, the population differentiation method can reject the neutral hypothesis and infer the presence of a selective effect. The Fst methods generally adopt the genome unit point scanning strategy. Fst can detect differences in allele frequencies between populations and effectively detect the selected site in the population [9]. The $\theta \pi$ ratio based on gene heterozygosity is also a common detection method. This is a parameter that measures the polymorphism of a specific population. The larger the $\pi$ value, the higher the polymorphism of its corresponding subgroup [10]. Li et al. [11] performed selection signal analysis (Fst and $\theta \pi$ ratio) on resequencing data from 70 cashmere and 14 non-cashmere goats, screening out several genes potentially involved in cashmere fiber formation fibroblast growthfactor $5(F G F 5)$, serine/threonine-protein kinase (SGK3), lin-like growth factor binding protein 7(IGFBP7), oxytocin receptor(OXTR), and rho associated coiled-coil containing protein kinase 1(ROCK1).

$\mathrm{XP}-\mathrm{EHH}$ is an extension of the statistical principles of EHH and His [12] and can be used to detect genome-wide selective roles in a given population. Zhong et al. [8] used the EHH method to detect the important core regions of 202 Jinhua pigs, and screened the genes related to economic traits such as meat quality, reproductive ability, immune response, and external traits, identifying the microRNA ssc-mir-365-2 and the lysine demethylase $8(K D M 8)$, rabaptin, RAB GTPase binding effector protein 2(RABEP2), GSG1 like(GSG1L), ras homolog, mTORC1 binding (RHEB), and rabphilin 3A like(RPH3AL) genes. Yin et al. [13] performed a genome-wide sequencing of six Pengxian yellow chickens and, through selective scanning analysis, detected several regions with strong selection signals, including 497 protein-coding genes. These genes were involved in developmental processes, metabolic processes, responses to external stimuli, as well as other biological processes, such as digestion ATP binding cassette subfamily $\mathrm{G}$ member 5(ABCG5), ATP binding cassette subfamily $\mathrm{G}$ member $8(A B C G 8)$ and adrenoceptor $\beta$ 1(ADRB1), muscle development and growth sphingomyelin phosphodiesterase 3(SMPD3), neural EGFL like 1(NELL1) and BicC family RNA binding protein 1(BICC1), and decreased immune function metastasis associated 1 family member 3(MTA3). Zhang et al. [14] successfully detected the whole-genome copy number variation (CNV) of 318 individuals from 24 Chinese native cattle breeds and 37 yaks as outgroups by analyzing the population structure and adaptability to high altitude. Although selective signatures have been successfully used to screen genes related to livestock economic traits, data are lacking about the sheep wool shedding. Here, we selected 27 sheep breeds in populations of two wool types and, using Illumina ovine SNP 50K chip typing data to analyze the selective signatures. Based on the Fst, $\theta \pi$ ratio, and XP-EHH results to screen candidate genes related to sheep 
hair follicle development and depilation traits, in order to provide a reference for sheep molecular breeding.

\section{Materials and Methods}

\subsection{Ethics Statement}

This study was conducted according to the guidelines for the care and use of laboratory animals promulgated by the State Council of China. This research was approved by the Animal Management and Ethics Committee of Lanzhou Institute of Animal Husbandry and Veterinary Medicine, Chinese Academy of Agricultural Sciences (license number: 2019-008).

\subsection{Sample and Data Processing}

In this study, a total of 795 individuals from 27 breeds of domestic and foreign sheep were selected for inclusion. The sheep were divided into fine-wool sheep and hair sheep, according to the wool type. The names, abbreviations, and sample size information for each variety are shown in Table 1 . The Illumina ovine SNP 50K chip genotyping data for the 27 breeds used in the study were obtained from the International Sheep Genomics Consortium (ISGC) (https://www.sheephapmap.org/, accessed on 13 June 2021), National Animal Genome Research Program (NRSP) (https:/ / www.animalgenome. $\mathrm{org} /$ sheep/community/, accessed on 25 June 2021), a Web-Interfaced next generation Database dedicated to genetic Diversity Exploration (WIDDE) (http:/ / widde.toulouse. inra.fr/, accessed on 9 July 2021), and the Ontario Sheep Farmers (OSF) (https: / / www. ontariosheep.org/, accessed on 21 July 2021). Because the 50K chip genotyping data in the databases uses different versions of the sheep reference genome, the SNP location information was reordered using the locations of the Ovis_aries_v4.0 reference genome. Quality control was performed with Plink (v1.90) [15] software. The quality control standard [16] was as follows: (1) The genotype information of the autosomal chromosomes 1-26 was extracted for subsequent analysis, with the parameter-chr1-26; (2) Sites with SNP deletion rates $>0.1$ were excluded, with the parameter-geno 0.1 ; (3) The minor allele frequency (MAF) was $>0.01$, with the parameter-maf 0.01. Finally, 37895 SNPs were obtained for further analysis.

\subsection{Population Structure}

Principal Component Analysis (PCA) is a dimensionality reduction statistical method aided by orthogonal transformation that recombines the original variables into a new set of independent comprehensive variables [17]. In population genetics, samples are clustered according to their principal components based on the degree of SNP differences in the individual genome and the characteristics of each trait. In the present study, PCA was conducted by using Plink (v1.90) software with the parameter plink-pca 3. The results were visually displayed using the ggplot package in R [18].

\subsection{Selective Signatures}

The combined methods of the Fst unit point, sliding window, and $\theta \pi$ ratio were used to analyze the selective signatures of sheep populations with different wool types. Data analysis was completed using Vcftools (0.1.15) [19] software. In brief, the Fst value in the chromosome was calculated with $500 \mathrm{~kb}$ as the sliding window and $50 \mathrm{~kb}$ as the step window $[20,21]$, followed by extraction of the sites in the window where the Fst and $\theta \pi$ ratio were in the top 5\% [22]. These sites were regarded as significant SNP candidate sites for the selective signatures. As the degree of linkage disequilibrium between sites is gradually reduced due to increasing marker spacing, the EHH of different lengths caused by selection can be observed in the genome. Although EHH can accurately select relevant genes under selection pressure, it does not distinguish whether these genes are newly mutated alleles or ancestral alleles [22]. Therefore, this study used the integrated haplotype score (iHS) test and the cross-population extended haplotype homozygosity (XP-EHH) 
test. The rehh package in $\mathrm{R}$ [23] was used for intra-group and inter-group selection signal detection. The ihh2ihs command in rehh was used to calculate the iHS value of the sheep population, after which the ies2xpehh command in rehh was used to calculate the XP-EHH value of the hair sheep, to detect the sites with different selection pressures between the hair sheep and fine-wool sheep [24].

Table 1. Information of the sheep populations used in this study.

\begin{tabular}{cccc}
\hline Breed & Abbreviation & Samples Size & Wool Type \\
\hline Arawapa & APA & 37 & fine-wool sheep \\
Australian Poll Merino & APM & 98 & fine-wool sheep \\
Brazilian Creole & BCS & 23 & fine-wool sheep \\
Chinese Merino & CME & 23 & fine-wool sheep \\
Meat Lacaune & LAC & 75 & fine-wool sheep \\
Australian Merino & MER & 88 & fine-wool sheep \\
Rambouillet & RMB & 102 & fine-wool sheep \\
SwissBlack-Brown Mountain Sheep & SBS & 24 & fine-wool sheep \\
African Dorper & ADP & 21 & hair sheep \\
Adane & AKD & 12 & hair sheep \\
Arabo & AKR & 10 & hair sheep \\
African White Dorper & AWD & 6 & hair sheep \\
Barbados Black Belly & BBB & 24 & hair sheep \\
Morada Nova & BMN & 22 & hair sheep \\
Bonga & BQ & 9 & hair sheep \\
Santa Ines & BSI & 47 & hair sheep \\
Doyogena & DA/DH & 15 & hair sheep \\
Kefis & FKD & 13 & hair sheep \\
Gesses & GGD & 11 & hair sheep \\
Hammari & H & 11 & hair sheep \\
Kabashi & K & 9 & hair sheep \\
Kidor & KO & 10 & hair sheep \\
Loya & LA & 15 & hair sheep \\
Molale & MZ & 15 & hair sheep \\
Red Maasai & RMA & 45 & hair sheep \\
Shubi Gemo & SHG & 15 & hair sheep \\
Gafera & WA & 15 & hair sheep \\
\hline
\end{tabular}

\subsection{Candidate Gene Detection and Annotation}

The relevant SNP information was obtained after the detection of the selection pressure sites for the hair sheep population. Regions $50 \mathrm{~kb}$ upstream and downstream of the SNP are considered as the selected regions of signal action [25], with reference to the Ovis_aries_v4.0 genome (https://www.ncbi.nlm.nih.gov/assembly/GCF_000298735.2, accessed on 3 August 2021) information annotating the regions of selective signatures.

\section{Results and Data Analyses}

\subsection{Analysis of the Population Structure}

The PCA of the genotyping data from the 27 breeds is shown in Figure 1. PC1 could explain $4.42 \%$ of the genetic variation, while PC2 could explain $1.74 \%$. Through PC1, PC2, and PC3, it was found that the sheep populations clustered together according to wool types, indicating that the genetic relationship between the two wool types is relatively distant. 


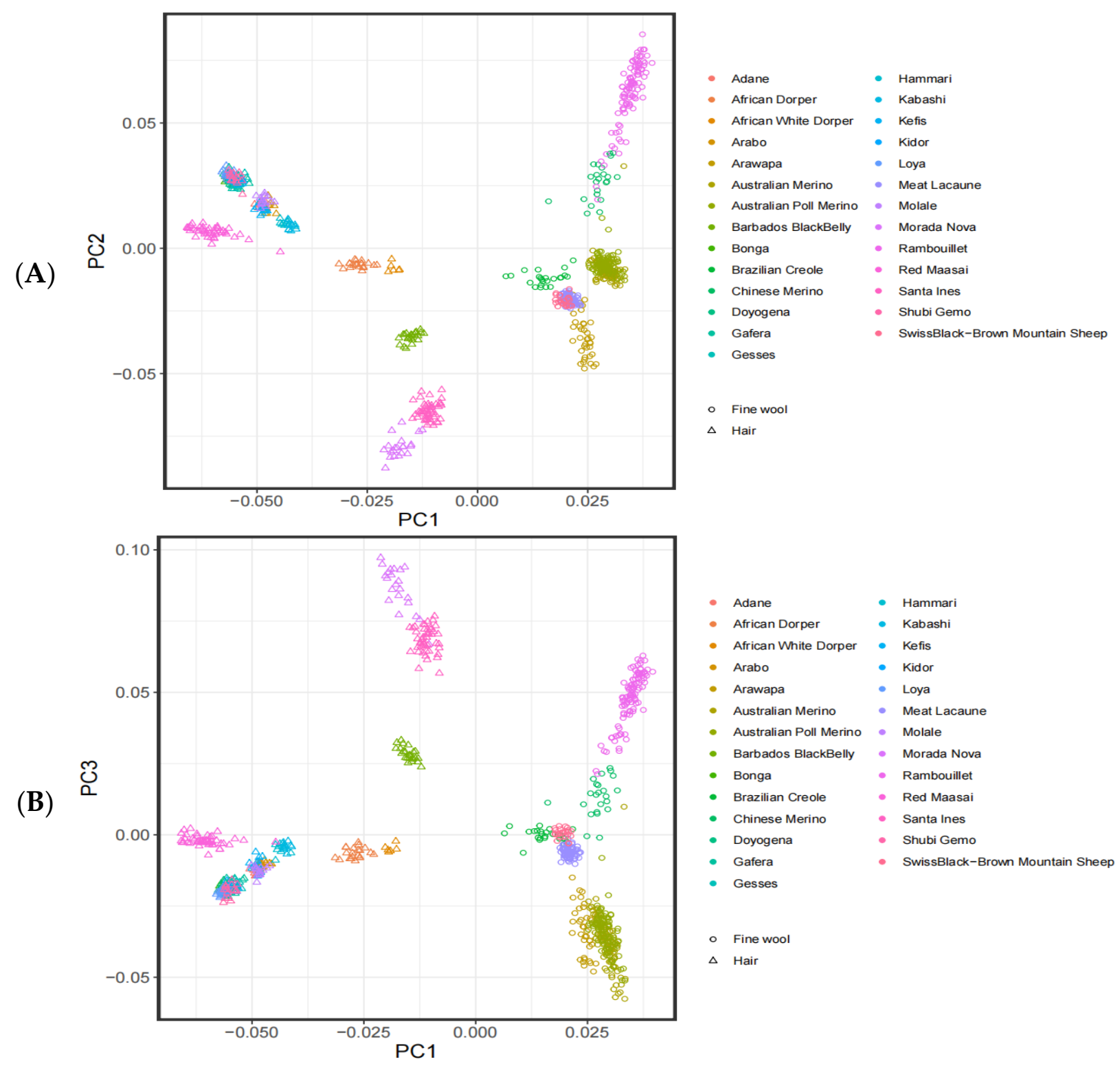

Figure 1. Principal component analysis of the different sheep populations. (A) Analysis results of PC1 and PC2 between the fine-wool sheep and hair sheep populations; (B) Analysis results of PC1 and PC3 between the fine-wool sheep and hair sheep populations.

\subsection{Fst and $\theta \pi$ Ratio}

Compared with the fine-wool sheep, shedding is characteristic of the hair sheep. It has obvious shedding characteristic and high anti-parasite. Although the main factors affecting wool shedding in this sheep are not clear, there is preliminary evidence that the shedding is strongly controlled by heredity, most of its expression is a single dominant autosomal gene. Through the detection and analysis of selective signatures between two wool-type sheep populations, the genomic regions affecting shedding in the hair sheep were identified. Fst and $\theta \pi$ ratio analysis of the hair sheep were referenced to the fine-wool sheep using Plink (v1.90) software to analyze the 37,895 SNP sites to obtain the pairwise genetic differentiation index Fst and the $\theta \pi$ ratio between two wool-type populations. At the genome-wide level, the top 5\% SNP sites identified by the Fst (Fst > 0.0536) and $\theta \pi$ ratio $(\theta \pi$ ratio $>1.1398)$ analysis were taken as candidate target sites. Among them, 1262 SNP sites were higher than the threshold line (Figure 2), including 110 sites at the tail of the Fst distribution (Fst $>0.25$ ) and 389 sites with large deviations $(\theta \pi$ ratio $>2$ ). 


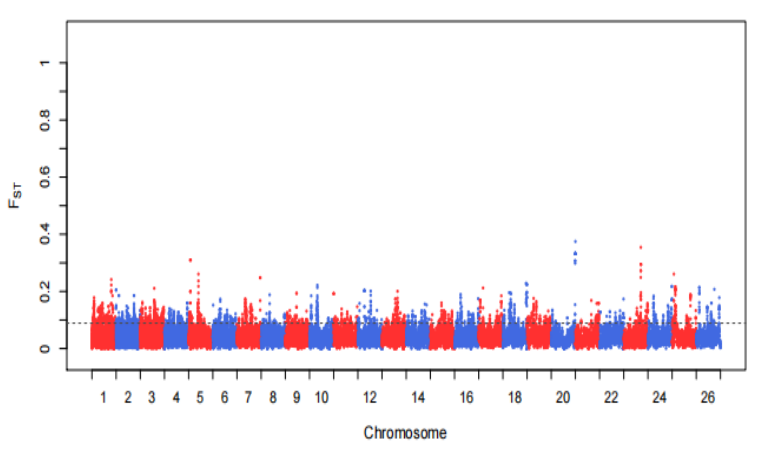

(A)

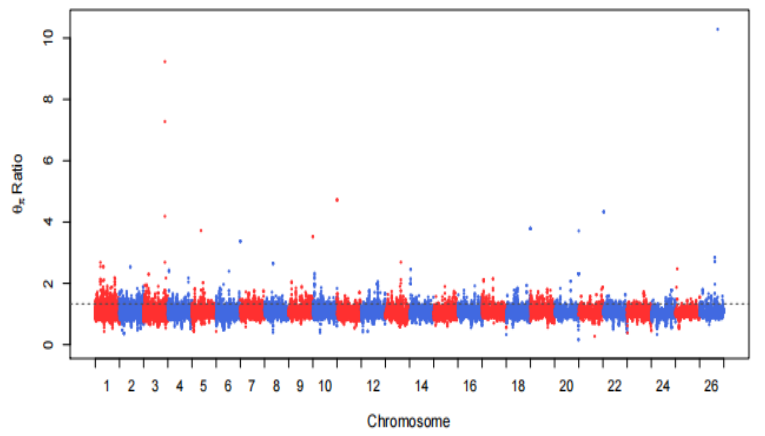

(B)

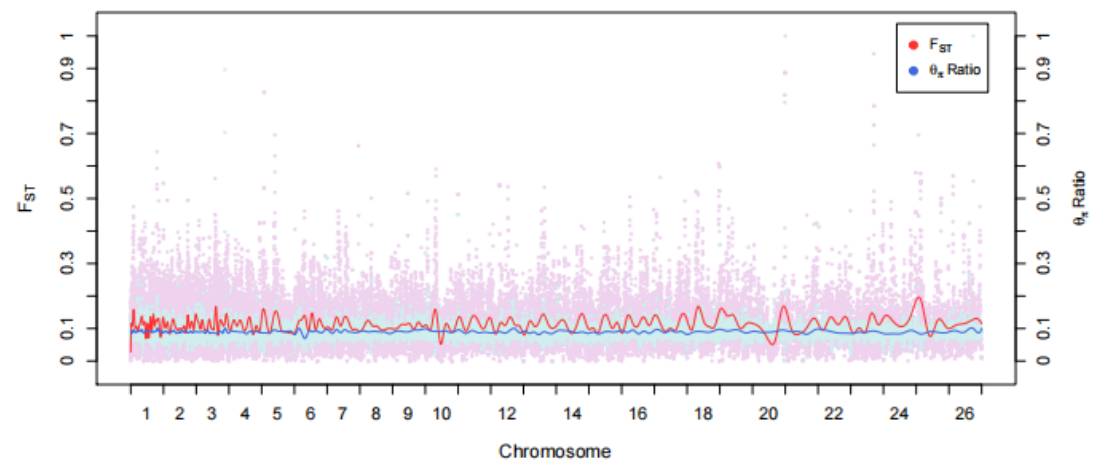

(C)

Figure 2. Analysis of selection signal distribution on sheep autosomes. (A) Manhattan map of Fst; (B) Manhattan map of $\theta \pi$ ratio; (C) Manhattan map of Fst and $\theta \pi$ ratio joint analysis.

\subsection{XP-EHH Analysis}

By comparing the differences in extended haplotype homozygosity between the two wool-type sheep populations, the genomic regions affecting shedding in the hair sheep were examined. The fine-wool sheep population was used as the reference population and the hair sheep as the experimental population for selection signal analysis. As shown in Figure 3, the sites with XP-EHH $<-2$ were selected as SNP sites in the hair sheep population [26]. In addition, a total of $618 \mathrm{SNP}$ sites were selected in the hair sheep population. The frequency distribution histogram (Figure 4) of the XP-EHH values of hair sheep showed that the XP-EHH between the hair and fine-wool sheep followed a normal distribution.

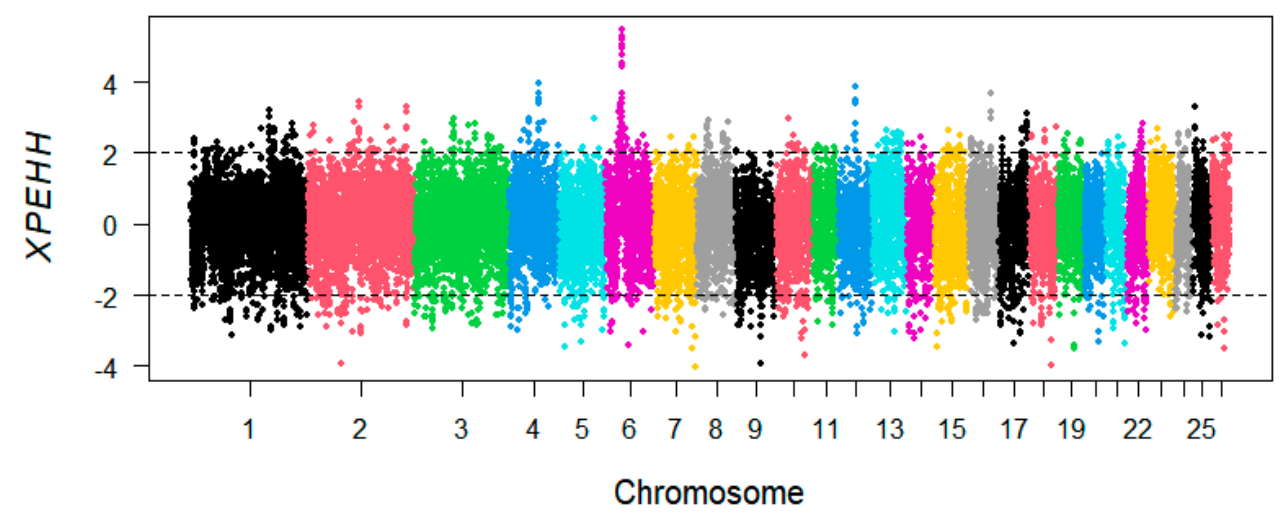

Figure 3. Analysis of selection signal distribution on sheep autosomes. Chromosomes 1 to 26 are shown in different colors; the horizontal dotted black line indicates the genome-wide significance level. 


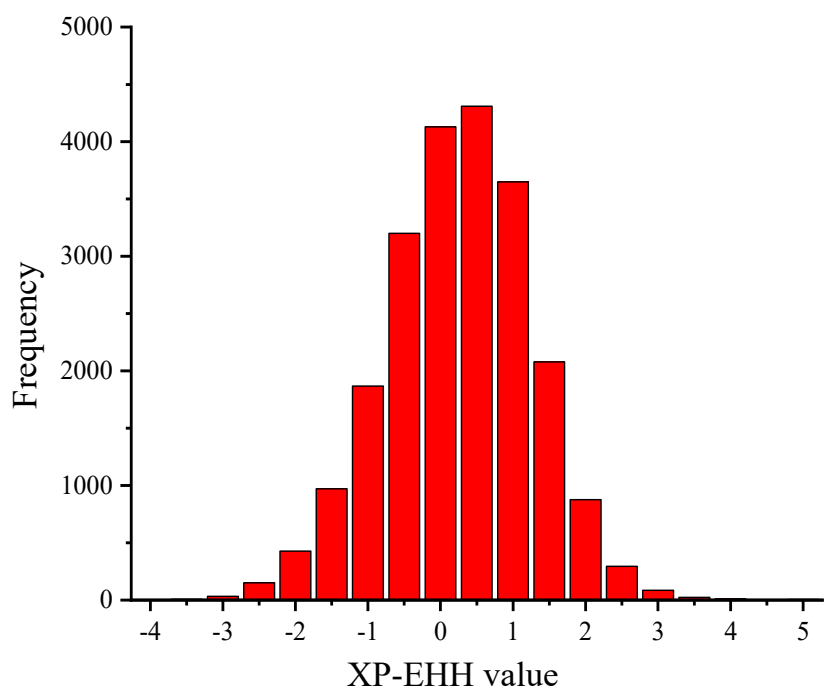

Figure 4. The distribution of XP-EHH in hair sheep and fine-wool sheep. The abscissa represents the haplotype value and the ordinate represents the number of occurrences of each region.

\subsection{Gene Mapping and Functional Annotation}

With reference to the sheep Ovis_aries_v4.0 genome information, the Fst, $\theta \pi$ ratio, and XP-EHH were used to screen the selected SNP sites in the hair sheep population for annotation. Of these, 1262 were identified in the combined Fst and $\theta \pi$ ratio analyses, and 618 sites in the XP-EHH analysis. Next, a Venn diagram was drawn to illustrate these results, which showed 115 SNP loci in the overlapping region (Figure 5). Interestingly, after gene mapping and functional annotation, a $\mathrm{G} \rightarrow \mathrm{T}$ mutation was detected at SNP site 41339449 in Chr5 at a distance of 2996 bp from TCF3, a C $\rightarrow$ T mutation was detected at SNP site 52044015 in Chr13 at a distance of 825 bp from TGM3, a G $\rightarrow$ A mutation was seen at SNP site 52055150 located within TGM3, a C $\rightarrow$ T mutation was observed at SNP site 53089456 in Chr13 at distance of 26,609 bp from SOX18, and a G $\rightarrow$ A mutation was detected at SNP site 48786396 in Chr14 located within PRX (Table 2). Deep analysis of the two SNPs sites, 13-52055150 and 14-48786396, were performed using MEGA (5.0) and BioEdte (7.0) software. The results showed that SNP site 52055150 in TGM3 was located in the non-coding region between exon 8 (52051614-52051922) and exon 9 (52055639-52055881), which does not participate in the transcription process and does not cause changes in amino acids and proteins. The SNP locus 14-48786396 within the PRX gene is in exon 7 and is involved in coding amino acid at position 387. This site changes the codon from AGC to AAC and is a missense mutation, resulting in the change of its encoded amino acid from aspartic acid (N) to serine (S). A single mutation at this site causes changes in its mRNA secondary structure, in turn affecting the structure of the protein.

Table 2. Candidate genes associated with hair follicle development and wool shedding traits in hair sheep.

\begin{tabular}{cccccc}
\hline Chromosome $^{\mathbf{1}}$ & Position (bp) $^{\mathbf{2}}$ & REF $^{\mathbf{3}}$ & ALT $^{\mathbf{4}}$ & Gene Name $^{\mathbf{5}}$ & Distance(bp) $^{\mathbf{6}}$ \\
\hline 5 & 41339449 & $\mathrm{G}$ & $\mathrm{T}$ & TCF3 & 2996 \\
13 & 52044015 & $\mathrm{C}$ & $\mathrm{T}$ & TGM3 & 825 \\
13 & 52055150 & $\mathrm{G}$ & $\mathrm{A}$ & $T G M 3$ & within \\
13 & 53089456 & $\mathrm{C}$ & $\mathrm{T}$ & $S O X 18$ & 26,609 \\
14 & 48786396 & $\mathrm{G}$ & $\mathrm{A}$ & PRX & within \\
\hline
\end{tabular}

${ }^{1}$ The Ovis aries chromosome number of each significant SNP. ${ }^{2}$ The positions of each significant SNP associated with wool-shedding traits in the Ovis aries Oar_4.0 assembly. ${ }^{3}$ The base in the reference genome corresponding to the mutation site. ${ }^{4}$ Observed base change. ${ }^{5}$ The nearest annotated gene to each significant SNP. ${ }^{6}$ Designation of SNPs as within a gene or at a distance (bp) from a gene region, according to the Ovis aries Oar_4.0 assembly (https:/ / www.ncbi.nlm.nih.gov/assembly/GCF_000298735.2, accessed on 16 September 2021). 


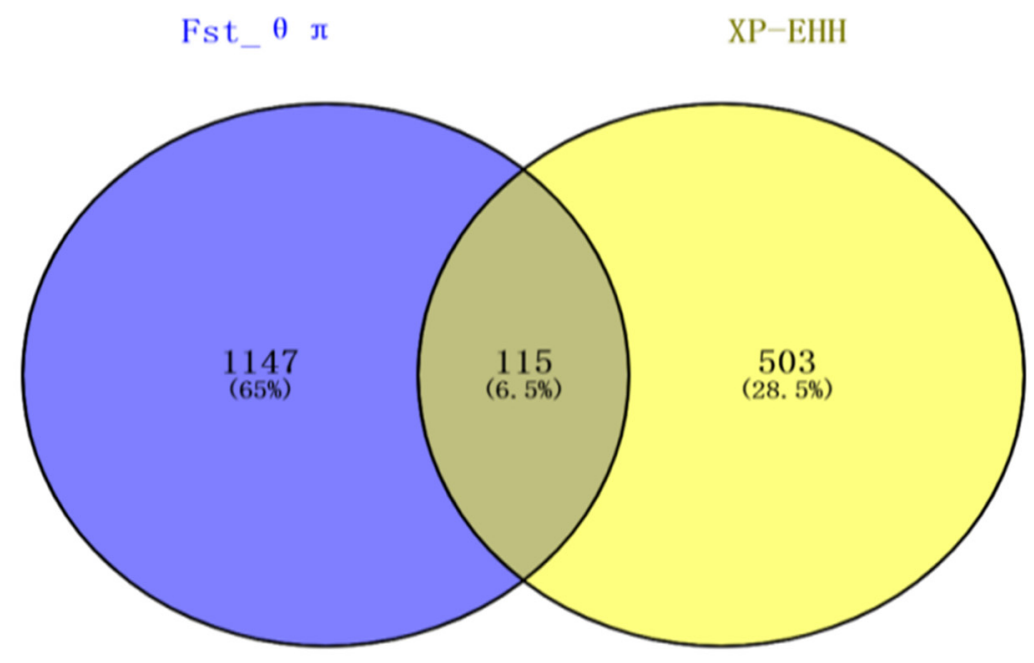

Figure 5. Overlapping SNP of Fst- $\theta \pi$ and XPEHH.

\section{Discussion}

In the current study, we selected two sheep populations with different wool types to investigate the clustering and genetic relationships of the selected samples through PCA. PCA is an unsupervised linear technique for dimension reduction and allows the extraction of axes of maximal variation from datasets [27]. This method was used to allocate them to groups using genotypic markers. When the classification is not obvious, and animals of the same breed tend to be located close together in PCA plots [28]. As it was assumed that some of the animals used in the current study may not have been pure-bred and unmanaged crossbreeding could have occurred in their herds, PCA was used to allocate them to groups using genotypic markers [29]. The results showed that the PC1 separated the 27 sheep breeds according to different wool types, including 470 finewool sheep and 325 hair sheep, with the clear separation between the fine-wool and hair sheep indicative of a relatively distant genetic relationship between the two populations. In contrast, PC2 and PC3 were distinct from each other in terms of the different varieties but the distance was small. In recent years, the analysis of selective signatures has become an important tool for screening the economic traits of animals and plants. Fst can scan for genome-wide SNP sites, calculating the Fst value for each SNP, using a range of values from 0 to 1 , where 0 represents no differentiation between the populations at any of the sites and 1 represents complete differentiation [30]. Fst values between $0.05-0.15$ indicate moderate genetic differentiation between populations, while values of $0.15-0.25$ suggest a large difference, with values over 0.25 indicative of substantial differentiation [30,31]. To improve the detection accuracy, the combination of unit point and sliding window is used to reduce the probability of false positives [32]. The $\theta \pi$ ratio is analyzed according to gene heterozygosity, and the ratio between the two populations is then calculated. The higher the degree of genome selection, the more the $\theta \pi$ ratio deviates from 1 [11]. XP-EHH is based on the principle of gene linkage disequilibrium. After manual selection and genetic improvement, a large amount of chromosomal recombination is apparent in a population. However, the presence of linkage results in the generation-to-generation transmission of neutral sites near the mutant gene, forming a long range of haplotype homozygosity on the chromosome. Compared with the Fst that can only calculate the site of differentiation, $\mathrm{XP}-\mathrm{EHH}$ statistics can further identify the population where the selection occurred. We used a combination of these methods together with mutual validation to improve the accuracy of gene localization, resulting in the identification of 115 SNP sites identified in the overlapping region between the three methods; of these, gene functional annotation yielded 106 significant genes, including 64 with known functions.

Based on the overlapping region, we identified four potential genes (PRX, SOX18, $T G M 3$, and TCF3) that were directly or indirectly associated with hair follicle development 
and wool shedding. Marker 14_48786396 on Chr14, associated with the skin and hair follicles, was located within PRX. Previous studies have demonstrated that members of the $P R X$ gene family encode peroxidases, which can utilize the activated cysteine to remove peroxides and hydroxyl radicals. The PRX homotype (I-VI) is related to cell proliferation, differentiation, and anti-apoptosis [33]. In terms of skin diseases, PRXII was found to be expressed in the vascular endothelial cells of normal skin [34]. In addition, in previous studies on the isoform-specific expression of peroxidases in rat epidermis and hair follicles, three PRX isoforms were identified, with PrxII located in the epidermal cells and epidermal accessory structures of the dermis (hair follicles, eccrine glands, and sebaceous glands) [35]. Primary cilia were observed in the hair follicle dermal papillae of Prx1-Cre skin in mice expressing Prx1-driven Cre recombinase [36]. These findings suggest that $P R X$ represents a highly significant gene in the determination of wool properties.

The SOX gene family encodes transcription factors. Its products have a HMG motif conserved region [37], that is involved in the regulation of embryonic development and the determination of cell fate. The encoded proteins act as transcriptional regulators in the development of hair, blood vessels, and lymphatic vessels through complexation with other proteins. SOX18 belongs to the SRY-related HMG domain family and is one of the important members of the SOX transcription factor F (SOX F) subfamily. It regulates angiogenesis [38], lymphangiogenesis [39], and hair follicle differentiation [40]. It is located in Chr20 and Chr2 in human and mouse, respectively, and plays an important role in the development of blood vessels and lymphatics [41]. It has been found that SOX18 is expressed in hair follicles during mouse embryonic development [40], and a SOX18 point mutation has been found in two different mutant mouse alleles. Compared with the wild-type, the fusion protein containing this mutation lacked the ability to activate transcription and it has been found that the mutation induces hair follicle defects in spontaneous mouse mutants [41], indicating that SOX18 plays a vital role in hair follicle development or function. Previous studies on the effects of SOX18 in endothelial cells found that the gene was transiently expressed in the mesenchyme of endothelial cells and hair follicles during mouse embryogenesis and played a key role in the development of hair follicles [42]. In the induction experiment of dermal papilla on hair follicle growth stage, it was found that SOX18 was specifically expressed in the dermal papillae of all dorsal hair follicle types [43]. A study on human patients and their offspring identified a recessive mutation in SOX18 that played a key role in the development of hair, blood vessels, and lymphatic vessels [44], and was mainly manifested clinically as sparse hairlymphedema-telangiectasia [39]. In summary, the abnormal expression of SOX18 can cause sparse hair-lymphedema-telangiectasia syndrome, and SOX18 has been identified as a key gene in hair follicle formation. The SNP locus located at 53089456 was detected on chr13, at a 26,609 bp distance from the SOX18 locus and was found to be strongly selected in the hair sheep population. Therefore, it is speculated that SOX18 plays a key role in sheep hair follicle formation. Although the abnormal expression of SOX18 may be related to sheep hair follicle development and depilation traits, this requires further verification.

Marker 13_52044015 and 13_52055150 on Chr13, were located beside ( 825 bp) and within TGM3, which encodes type 3 glutamine transaminase (tgase-3) [45], an enzyme with $\mathrm{Ca}^{2+}$-dependent transaminase activity in epidermal non-proliferative layers and hair follicles [46]. Mutation of human TGM1 leads to type I autosomal recessive lamellar ichthyosis, which is characterized by skin surface squamous epidermal hyperplasia and barrier defects [47]. On the other hand, TGM3 knockdown in mice resulted in developmental delays in skin barrier formation in utero, with abnormalities in hair follicle function [48]. It is thus inferred that TGM3 is closely related to the development of hair follicles, although its mechanism of action requires further research.

Hair follicle stem cells are mainly located in the bulge of the outer root sheath of the hair follicles. They belong to the adult stem cell, which can differentiate into epidermis, hair follicles, and sebaceous glands, and participate in the process of skin wound healing [49]. For example, in human skin, hair follicle pluripotent stem cells are considered to be 
necessary for the hair cycle and epidermal wound repair [50], and TCF3 is naturally expressed in hair follicle pluripotent stem cells. During the growth phase of the hair cycle, TCF3 is expressed not only in quiescent pluripotent stem cells but also in the outer basement layer of the newly formed outer root sheath [51]. A study found the Tcf protein not only plays a role in Wnt signaling, but also acts as an inhibitor when $\beta$-catenin is low or absent. Moreover, Tcf3 and Tcf4 may have wnt-dependent and wnt-independent effects, which are essential for the establishment and maintenance of all skin epithelial stem cells [52]. When the level of Tcf3/4-Tle is high, hair follicle stem cells can maintain their characteristics, but still static. When Tcf3/4-Tle levels drop or Wnt- $\beta$-catenin levels rise, this balance changes and hair regeneration begins [53]. In addition, the TCF3 suppressor gene includes transcription regulators of the epidermal, sebaceous gland, and hair follicle differentiation program [50], regulates cell proliferation and apoptosis, induces hair follicle stem cell differentiation, and promotes the transformation of hair follicles from the resting phase to the growth phase [54]. These results suggest that TCF3 plays a vital role in hair follicle stem cells and the Wnt pathway and its associated signal transduction.

\section{Conclusions}

The current study used Fst, the $\theta \pi$ ratio, and XP-EHH to detect the selective signatures of sheep SNP chip typing data. A total of 115 SNPs sites were identified, of which the PRX, SOX18, TGM3, and TCF3 genes were found to be closely related to hair follicle formation, epidermal differentiation, hair follicle stem cell development, and wool shedding, located on Chr5, Chr13, and Chr14. These findings provide a reference for the study of the mechanism of sheep hair follicle development. In future studies, we will use high-density chips and an increased number of populations to study these genes and their functions in hair development in greater depth.

Author Contributions: Conceptualization, Y.Y., B.Y. and L.Z.; methodology, Z.L. (Zhihui Lei), T.G. and H.Z.; software, Z.L. (Zhihui Lei), S.Z. and C.Y.; validation, Z.L. (Zengkui Lu) and G.Q.; formal analysis, Z.L. (Zhihui Lei); investigation, Z.L. (Zhihui Lei) and W.S.; resources, Y.Y. and J.L. (Jianbin Liu); data curation, W.S. and M.H.; writing_-original draft preparation, Z.L. (Zhihui Lei); writing - review and editing, Z.L. (Zhihui Lei) and W.S.; visualization, Z.L. (Zhihui Lei); supervision, Y.Y. and J.L. (Jianye Li); project administration, Y.Y. and B.Y. All authors have read and agreed to the published version of the manuscript.

Funding: This research including experimental design, sample collection, data analysis, and manuscript writing, was funded by the fine-wool sheep resources and breeding innovation team of the Science and Technology Innovation Project of the Chinese Academy of Agricultural Sciences (25-LZIHPS-07); the major scientific research task of the Science and Technology Innovation Project of the Chinese Academy of Agricultural Sciences "The cultivation and industrialization of new breeds of high-quality and efficient mutton sheep" (CAAS-ZDRW202106) and National Wool Sheep Industry Technology System Breeding Technology and Method Post (CARS-39-02).

Institutional Review Board Statement: The study was conducted according to the guidelines of the Declaration of Helsinki, and approved by the Institutional Review Board (or Ethics Committee) of Lanzhou Institute of Husbandry and Pharmaceutical Sciences of Chinese Academy of Agricultural Sciences (protocol code 2019-008 and 29 November 2019).

Informed Consent Statement: Not applicable.

Data Availability Statement: Not applicable.

Acknowledgments: We are thankful to all lab members for their support during the study.

Conflicts of Interest: The authors declare no conflict of interest. 


\section{References}

1. Jurado, N.V.; Leymaster, K.A.; Kuehn, L.A. Genetic Analysis of Wool Shedding Scores of Ewes from a Composite Flock using a Threshold Model and Bayesian Methodologies. In Proceedings of the 10th World Congress on Genetics Applied to Livestock Production, Vancouver, BC, Canada, 17-22 August 2014.

2. Pollott, G.E. A suggested mode of inheritance for wool shedding in sheep1. J. Anim. Sci. 2011, 89, 2316-2325. [CrossRef]

3. Cloete, S.; Snyman, M.; Herselman, M. Productive performance of Dorper sheep. Small Rumin. Res. 2000, 36, 119-135. [CrossRef]

4. Rose, G.; Mulder, H.; Thompson, A.; Van Der Werf, J.; Van Arendonk, J. Varying pasture growth and commodity prices change the value of traits in sheep breeding objectives. Agric. Syst. 2014, 131, 94-104. [CrossRef]

5. Matika, O.; Bishop, S.C.; Pong-Wong, R.; Riggio, V.; Headon, D.J. Genetic factors controlling wool shedding in a composite Easycare sheep flock. Anim. Genet. 2013, 44, 742-749. [CrossRef] [PubMed]

6. Combs, W. A History of the Barbados Blackbelly Sheep, 1st ed.; Westview Press: Boulder, CO, USA, 1983; pp. $179-197$.

7. Almeid, A. Barbados Blackbelly: The Caribbean ovine genetic resource. Trop. Anim. Health Prod. 2018, 50, 239-250. [CrossRef]

8. Zhong, X.; Hao, S.; Zhe, Z.; Zhao, Q.B.; Olasege, B.S.; Li, Q.M.; Yue, Y. Genome-wide detection of selective signatures in a Jinhua pig population. J. Integr. Agric. 2020, 19, 1314-1322.

9. Yang, F.-Y.; Guo, J.-J.; Liu, N.; Zhang, R.-Z. Genetic structure of the invasive Colorado potato beetle Leptinotarsa decemlineata populations in China. J. Integr. Agric. 2020, 19, 350-359. [CrossRef]

10. Ji, Y.S.; Wang, C.Y.; Liu, R. Exploitation of freezing-tolerant genes in pea (Pisum sativum L.) based on selective sweeping analysis. China Veg. 2020, 3, 33-42. (In Chinese)

11. Li, X.; Su, R.; Wan, W.; Zhang, W.; Jiang, H.; Qiao, X.; Fan, Y.; Zhang, Y.; Wang, R.; Liu, Z.; et al. Identification of selection signals by large-scale whole-genome resequencing of cashmere goats. Sci. Rep. 2017, 7, 15142. [CrossRef]

12. Zhao, F.-P.; Wei, C.-H.; Zhang, L.; Liu, J.-S.; Wang, G.-K.; Zeng, T.; DU, L.-X. A genome scan of recent positive selection signatures in three sheep populations. J. Integr. Agric. 2016, 15, 162-174. [CrossRef]

13. Yin, H.; Li, D.; Wang, Y.; Zhu, Q. Whole-genome resequencing analysis of Pengxian Yellow Chicken to identify genome-wide SNPs and signatures of selection. 3 Biotech 2019, 9, 383. [CrossRef]

14. Zhang, Y.; Hu, Y.; Wang, X.; Jiang, Q.; Zhao, H.; Wang, J.; Ju, Z.; Yang, L.; Gao, Y.; Wei, X.; et al. Population Structure, and Selection Signatures Underlying High-Altitude Adaptation Inferred From Genome-Wide Copy Number Variations in Chinese Indigenous Cattle. Front. Genet. 2020, 10, 1404. [CrossRef] [PubMed]

15. Purcell, S.; Neale, B.; Todd-Brown, K.; Thomas, L.; Ferreira, M.A.R.; Bender, D.; Maller, J.; Sklar, P.; de Bakker, P.I.W.; Daly, M.J.; et al. PLINK: A Tool Set for Whole-Genome Association and Population-Based Linkage Analyses. Am. J. Hum. Genet. 2007, 81, 559-575. [CrossRef] [PubMed]

16. Yuan, Z.H. Analysis of Selection Signatures Identify Genes Associated with Tail Type in Sheep. Anim. Genet. 2016, 48, 55-56. [CrossRef] [PubMed]

17. Sankhyan, V.; Thakur, Y.P.; Katoch, S.; Dogra, P.K.; Thakur, R. Morphological structuring using principal component analysis of Rampur-Bushair sheep under transhumance production in western Himalayan region, India. Indian J. Anim. Res. 2017. [CrossRef]

18. Wickham, H. Ggplot2: Elegant Graphics for Data Analysis; Springer Publishing Company, Incorporated: Berlin/Heidelberg, Germany, 2009.

19. Danecek, P.; Auton, A.; Abecasis, G.; Albers, C.A.; Banks, E.; DePristo, M.A.; Handsaker, R.E.; Lunter, G.; Marth, G.T.; Sherry, S.T.; et al. The variant call format and VCFtools. Bioinformatics 2011, 27, 2156-2158. [CrossRef]

20. Zeng, L.; Liu, H.-Q.; Tu, X.-L.; Ji, C.-M.; Gou, X.; Esmailizadeh, A.; Wang, S.; Wang, M.-S.; Wang, M.-C.; Li, X.-L.; et al. Genomes reveal selective sweeps in kiang and donkey for high-altitude adaptation. Zool. Res. 2021, 42, 450-460. [CrossRef]

21. Wu, D.-D.; Yang, C.-P.; Wang, M.-S.; Dong, K.-Z.; Yan, D.-W.; Hao, Z.-Q.; Fan, S.-Q.; Chu, S.-Z.; Shen, Q.-S.; Jiang, L.-P.; et al. Convergent genomic signatures of high-altitude adaptation among domestic mammals. Natl. Sci. Rev. 2019, 7, 952-963. [CrossRef]

22. Abied, A. Genome-wide Characterization of the Sudanese and Chinese Indigenous sheep populations by High Density SNP arrays. Ph.D. Thesis, Chinese Academy of Agricultural Sciences, Beijing, China, 2020.

23. Gautier, M.; Klassmann, A.; Vitalis, R. REHH 2.0: A reimplementation of the R package REHH to detect positive selection from haplotype structure. Mol. Ecol. Resour. 2017, 17, 78-90. [CrossRef]

24. Ye, R.; Tian, Y.; Huang, Y.; Zhang, Y.; Wang, J.; Sun, X.; Zhou, H.; Zhang, D.; Pan, W. Genome-wide Population Genetics Analysis of Plasmodium fallciparum Isolates from China-Myanmar border. Frontiers 2019. [CrossRef]

25. Yan, J.; Zou, D.; Li, C.; Zhang, Z.; Song, S.; Wang, X. SR4R: An Integrative SNP Resource for Genomic Breeding and Population Research in Rice. Genom. Proteom. Bioinform. 2020, 18, 173-185. [CrossRef] [PubMed]

26. Kiptoo, C.E.; Cheruiyot, B.R.; Oluoch, A.J. Signatures of Selection in Admixed Dairy Cattle in Tanzania. Front. Genet. 2018, 9, 607.

27. Jolliffe, I.T.; Cadima, J. Principal component analysis: A review and recent developments. Philos. Trans. R. Soc. A Math. Phys. Eng. Sci. 2016, 374, 20150202. [CrossRef]

28. Manel, S.; Gaggiotti, O.; Waples, R.S. Assignment methods: Matching biological questions with appropriate techniques. Trends Ecol. Evol. 2005, 20, 136-142. [CrossRef]

29. Moradi, M.H.; Khaltabadi-Farahani, A.H.; Khodaei-Motlagh, M.; Kazemi-Bonchenari, M.; McEwan, J. Genome-wide selection of discriminant SNP markers for breed assignment in indigenous sheep breeds. Ann. Anim. Sci. 2021, 21. [CrossRef]

30. Wright, S. The Genetical Structure of Populations. Ann. Eugenic. 1950, 15, 323-354. [CrossRef] 
31. Manzari, Z.; Mehrabani, H.; Nejati, A.; Moradi, H.; Gholizadeh, M. Detecting selection signatures in three Iranian sheep breeds. Anim. Genet. 2019, 50, 298-302. [CrossRef]

32. Ma, Y.; Ding, X.; Qanbari, S.; Weigend, S.; Zhang, Q.; Simianer, H. Properties of different selection signature statistics and a new strategy for combining them. Heredity 2015, 115, 426-436. [CrossRef]

33. Lee, S.-C.; Na, Y.-P.; Lee, J.-B. Expression of peroxiredoxin II in vascular tumors of the skin: A novel vascular marker of endothelial cells. J. Am. Acad. Dermatol. 2003, 49, 487-491. [CrossRef]

34. Lee, J.; Yun, S.; Chae, H.; Won, Y.; Kim, Y.; Lee, S.-C. Expression of peroxiredoxin and thioredoxin in dermatological disorders. Br. J. Dermatol. 2002, 146, 710-712. [CrossRef]

35. Lee, J.E.; Kwon, B.D.; Lee, J.-B.; Won, Y.-H.; Kim, Y.P.; Lee, S.-C.; Chae, H.Z.; Ahn, K.Y. Peroxiredoxin is Ubiquitously Expressed in Rat Skin: Isotype-Specific Expression in the Epidermis and Hair Follicle. J. Investig. Dermatol. 2000, 115, 1108-1114. [CrossRef]

36. DeRouen, M.C.; Zhen, H.; Tan, S.H.; Williams, S.; Marinkovich, M.P.; Oro, A.E. Laminin-511 and integrin beta-1 in hair follicle development and basal cell carcinoma formation. BMC Dev. Biol. 2010, 10, 112. [CrossRef] [PubMed]

37. Valenzuela, I.; Fernadez, P.; Plaja, A. Further delineation of the SOX18-related Hypotrichosis, Lymphedema, Telangiectasia syndrome (HTLS). Eur. J. Med. Genet. 2018, 3, 269-272. [CrossRef] [PubMed]

38. Duong, T.; Koltowska, K.; Pichol-Thievend, C.; Le Guen, L.; Fontaine, F.; Smith, K.A.; Truong, V.; Skoczylas, R.; Stacker, S.A.; Achen, M.G.; et al. VEGFD regulates blood vascular development by modulating SOX18 activity. Blood 2014, 123, 1102-1112. [CrossRef]

39. Francois, M.; Caprini, A.; Hosking, B.; Orsenigo, F.; Wilhelm, D.; Browne, C.; Paavonen, K.; Karnezis, T.; Shayan, R.; Downes, M.; et al. Sox18 induces development of the lymphatic vasculature in mice. Nature 2008, 456, 643-647. [CrossRef] [PubMed]

40. Pennisi, D.; Gardner, J.; Chambers, D.; Hosking, B.; Peters, J.; Muscat, G.; Abbott, C.; Koopman, P. Mutations in Sox18 underlie cardiovascular and hair follicle defects in ragged mice. Nat. Genet. 2000, 24, 434-437. [CrossRef] [PubMed]

41. Zhu, F.H.; Shi, J.Q. Research progress of transcription factor SOX18. J. Jining Med. Univ. 2016, 39, 280-282. (In Chinese)

42. Olsson, J.; Kamachi, Y.; Penning, S.; Muscat, G.; Kondoh, H.; Koopman, P. Sox18 expression in blood vessels and feather buds during chicken embryogenesis. Gene 2001, 271, 151-158. [CrossRef]

43. Hosking, B.M.; Wang, S.-C.; Chen, S.L.; Penning, S.; Koopman, P.; Muscat, G.E. SOX18 Directly Interacts with MEF2C in Endothelial Cells. Biochem. Biophys. Res. Commun. 2001, 287, 493-500. [CrossRef]

44. Legrand, J.M.; Roy, E.; Ellis, J.J.; Francois, M.; Brooks, A.J.; Khosrotehrani, K. STAT5 Activation in the Dermal Papilla Is Important for Hair Follicle Growth Phase Induction. J. Investig. Dermatol. 2016, 136, 1781-1791. [CrossRef]

45. Yamane, A.; Fukui, M.; Sugimura, Y.; Itoh, M.; Alea, M.P.; Thomas, V.; El Alaoui, S.; Akiyama, M.; Hitomi, K. Identification of a preferred substrate peptide for transglutaminase 3 and detection of in situ activity in skin and hair follicles. FEBS J. 2010, 277, 3564-3574. [CrossRef] [PubMed]

46. Schmuth, M.; Gruber, R.; Elias, P.M.; Williams, M.L. Ichthyosis Update: Towards a Function-Driven Model of Pathogenesis of the Disorders of Cornification and the Role of Corneocyte Proteins in These Disorders. Adv. Dermatol. 2007, 23, 231-256. [CrossRef] [PubMed]

47. John, S.; Thiebach, L.; Frie, C. Epidermal transglutaminase (TGase 3) is required for proper hair development, but not the formation of the epidermal barrier. PLoS ONE 2017, 7, e34252. [CrossRef] [PubMed]

48. Zheng, X.; Ni, Y.M.; Xie, S.J. The characteristics of hair follicle stem cells and their application in skin repair. Tradit. Chin. Med. 2015, 27, 73-75.

49. Nguyen, H.; Rendl, M.; Fuchs, E. Tcf3 Governs Stem Cell Features and Represses Cell Fate Determination in Skin. Cell 2006, 127, 171-183. [CrossRef] [PubMed]

50. Dasgupta, R.; Fuchs, E. Multiple roles for activated LEF/TCF transcription complexes during hair follicle development and differentiation. Development 1999, 126, 4557-4568. [CrossRef]

51. Lien, W.-H.; Polak, L.; Lin, M.; Lay, K.; Zheng, D.; Fuchs, E. In Vivo transcriptional governance of hair follicle stem cells by canonical Wnt regulators. Nature 2014, 16, 179-190. [CrossRef]

52. Nguyen, H.; Merrill, B.J.; Polak, L.; Nikolova, M.; Rendl, M.; Shaver, T.M.; Pasolli, H.A.; Fuchs, E. Tcf3 and Tcf4 are essential for long-term homeostasis of skin epithelia. Nat. Genet. 2009, 41, 1068-1075. [CrossRef] [PubMed]

53. Wu, C.I.; Hoffman, J.A.; Shy, B.R. Function of Wnt/ $\beta$-catenin in counteracting Tcf3 repression through the Tcf3- $\beta$-catenin interaction. Development 2012, 139, 2118-2129. [CrossRef]

54. Luo, M.; Li, J.-F.; Yang, Q.; Zhang, K.; Wang, Z.-W.; Zheng, S.; Zhou, J.-J. Stem cell quiescence and its clinical relevance. World J. Stem Cells 2020, 12, 1307-1326. [CrossRef] 